\title{
A splice variant of human Bmal1 acts as a negative regulator of the molecular circadian clock
}

\author{
Jiwon Lee ${ }^{1}$, Eonyoung Park ${ }^{2}$, Ga Hye Kim ${ }^{1}, \|$ min Kwon ${ }^{1}$ and Kyungjin Kim ${ }^{3}$
}

\begin{abstract}
Bmal1 is one of the key molecules that controls the mammalian molecular clock. In humans, two isoforms of Bmal1 are generated by alternative RNA splicing. Unlike the extensively studied hBmal1b, the canonical form of Bmal1 in most species, the expression and/or function of another human-specific isoform, hBmalla, are poorly understood. Due to the lack of the N-terminal nuclear localization signal (NLS), hBMAL1a does not enter the nucleus as hBMAL1b does. However, despite the lack of the NLS, hBMAL1a still dimerizes with either hCLOCK or hBMAL1b and thereby promotes cytoplasmic retention or protein degradation, respectively. Consequently, hBMAL1a interferes with hCLOCK:hBMAL1binduced transcriptional activation and the circadian oscillation of Period2. Moreover, when the expression of endogenous $h B$ malla is aborted by CRISPR/Cas9-mediated knockout, the rhythmic expression of $h$ Per 2 and $h B m a l l b$ is restored in cultured HeLa cells. Together, these results suggest a role for hBMAL1a as a negative regulator of the mammalian molecular clock.
\end{abstract}

\section{Introduction}

The regulation of temporal and spatial coordination of gene expression is crucial for living systems. It involves the appropriate activation and/or repression of specific genes $^{1}$. The circadian clock is one of the most wellcoordinated biological systems that controls daily oscillations in physiology and behavior. At the molecular level, several core clock genes constitute interlocking feedback loops that drive the rhythmic transcription and translation of the core molecular clock components in the circadian clock system ${ }^{2,3}$. Briefly, heterodimers of CLOCK and BMAL1 (CLOCK:BMAL1) function as transcriptional activators and induce the rhythmic expression of downstream circadian clock genes such as Periods (Pers) and Cryptochromes $(\mathrm{Crys})^{4-6}$. Once synthesized in the

Correspondence: IImin Kwon (ilmin.kwon@skku.edu) or Kyungjin Kim (kyungjin@dgist.ac.kr)

${ }^{1}$ Department of Anatomy and Cell Biology, Sungkyunkwan University School of Medicine, Suwon 16419, Korea

${ }^{2}$ GenWay Biotech, Inc., 6777 Nancy Ridge Dr, San Diego, CA 92121, USA

Full list of author information is available at the end of the article. cytoplasm, Period and Cryptochrome proteins form a complex with Casein kinase I (CKI) and translocate to the nucleus to constitute the negative feedback loop by repressing their own transcriptional activation by CLOCK:BMAL1 heterodimers. Other downstream products of the CLOCK:BMAL1 complex, Rev-erbo and Rora, constitute additional feedback loops by repressing or activating the transcription of Bmal1, respectively ${ }^{3,7}$. These autoregulatory feedback mechanisms of core clock components serve as a fundamental driving force that generates and maintains the circadian rhythm. In addition to the core molecular feedback loops, increasing evidence suggests that post-translational modifications such as phosphorylation, sumoylation, and ubiquitination are closely involved in the fine-tuning of circadian clock control $^{3,8-12}$.

Along with post-translational modifications, alternative RNA splicing is also implicated in the regulation of the molecular circadian clock. For instance, in Neurospora crassa, loss of the splicing or mis-splicing of the White collar-2 gene leads to the production of a nonfunctional 
a
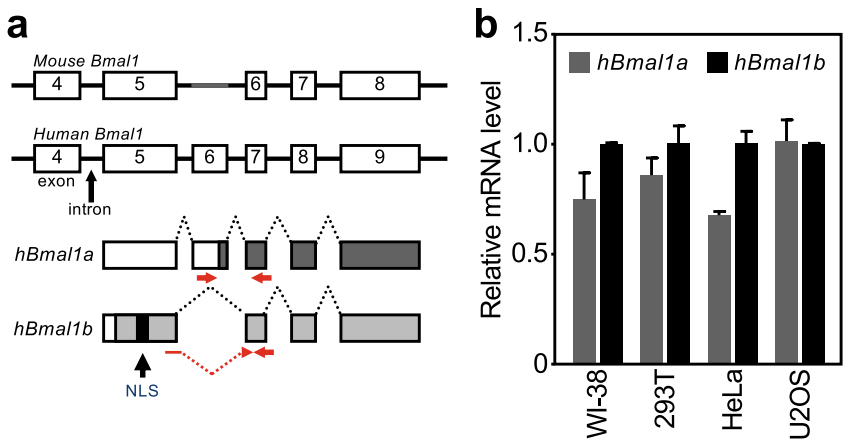

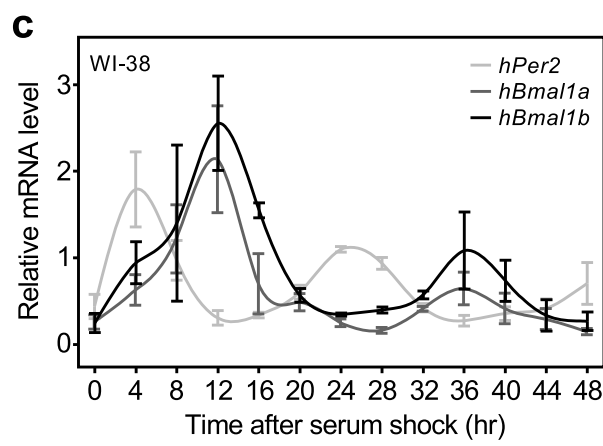

f d

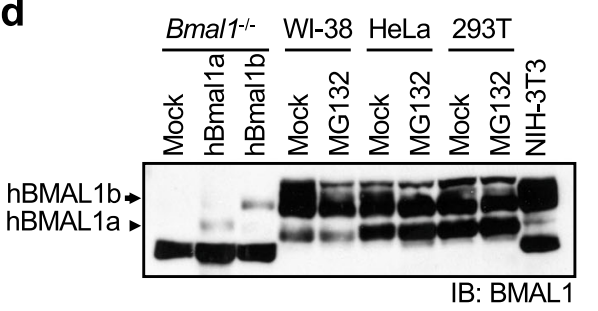

e

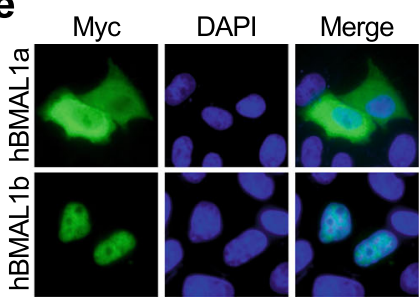

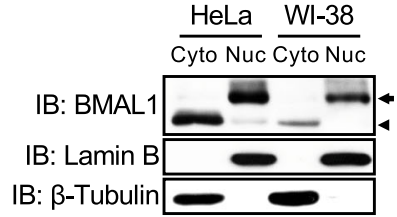

Fig. 1 Expression of human Bmal1 splice variants. a Schematic of the human and mouse Bmall genes. $\mathbf{b}$ Expression of $h B m a / 1 a$ and $h B m a / 1 b$ mRNAs was analyzed by qPCR in various human cell lines. c Circadian expression profiles of $h B$ mal1a (dark gray line), $h B m a l 1 b$ (black line), and $h P e r 2$ mRNAs (light gray line) were analyzed in human fibroblasts, WI-38 cells, by qPCR. $\mathbf{d}$ Expression of the endogenous hBMAL1a and hBMAL $1 \mathrm{~b}$ proteins was analyzed in several human and mouse cell lines by western blotting. For a size reference, exogenous hBMAL1a or hBMAL1b was expressed in $\mathrm{Bmal1}^{-1-}$ mouse embryonic fibroblasts (MEFs). e The subcellular localization of Myc-tagged hBMAL1a or hBMAL1b was observed under a fluorescence microscope after immunofluorescence staining using an anti-Myc antibody. $\mathbf{f}$ Expression levels of endogenous hBMAL1a (arrowhead) and hBMAL1b (arrow) in nuclear (Nuc) or cytoplasmic (Cyto) fractions of HeLa or WI-38 cells were investigated by western blotting. Subcellular fractionation was validated by western blotting using anti- $\beta$-Tubulin (cytoplasm) or anti-Lamin B (nucleus) antibodies

protein and subsequent arrhythmicity ${ }^{13}$. In addition, temperature-dependent splicing of another Neurospora core clock gene, Frequency, is reported to be implicated in the generation of circadian rhythms in $N$. crassa $^{14,15}$. In Drosophila melanogaster, flies containing a mutation at the splice site of exon 2 in Clock gene exhibit abnormal circadian rhythms ${ }^{16}$. In the Drosophila Period gene, the thermosensitive splicing of the intron in the 3 '-untranslated region (UTR) is critical for the flies to seasonally adapt to cold temperature ${ }^{17}$.

In mammals, different splice variants of Bmal1 are generated by alternative RNA splicing in humans, mice, and rats ${ }^{18-20}$. Based on an analysis of the sequences of different Bmal1 variants produced in three different species, we noticed that the human Bmalla isoform (hBmal1a) was the only variant in which the $\mathrm{N}$ terminal 43 amino acids are missing (Supplementary Fig. S1a). When exon 6 of the human Bmal1 gene is removed by RNA splicing, the canonical isoform $h B m a l 1 b$ is generated from the translational start signal located in exon 5 (Fig. 1a). However, when exon 6 is retained, the synthesis of hBmallb is terminated by the premature stop codon in exon 6 and another translation begins at the alternative initiation codon in the same exon to produce hBmalla without the $\mathrm{N}$-terminal region (Fig. 1a).
We began to focus on hBmalla because the nuclear localization signal (NLS) resides in the $\mathrm{N}$-terminal region that is absent in hBmalla (Supplementary Fig. S1a). Moreover, in the previous studies, it was reported that the NLS of Bmal1 is required for the nuclear localization of BMAL1 and its binding partner, CLOCK, and subsequent transcriptional activation of core clock genes by CLOCK: BMAL1 heterodimers in the nucleus ${ }^{21,22}$. The alternative RNA splicing of human Bmal1 and production of hBmal1a was first reported more than 20 years ago ${ }^{18}$. Bmal1 is a master transcriptional regulator of the mammalian molecular clock. However, little information is available about the functional implications of different kinds of Bmal1 variants in the regulation of the mammalian circadian clock. Here, we investigate for the first time the functional role of the human-specific Bmal1 variant, hBMAL1a, in the control of the molecular circadian clock.

\section{Materials and methods}

\section{Cell culture and transient transfection}

WI-38, HEK293T, HeLa, and NIH-3T3 cells were grown in Dulbecco's modification of Eagle medium supplemented with $10 \%$ fetal bovine serum (Thermo Fisher Scientific, Waltham, MA, USA) and $1 \%$ penicillin-streptomycin (Thermo Fisher Scientific) at 
$37^{\circ} \mathrm{C}$. Transient transfection was performed using Lipofectamine 2000 reagent (Thermo Fisher Scientific), according to the manufacturer's protocol.

\section{RT-PCR and $q P C R$}

Total RNA was isolated using Trizol (Thermo Fisher Scientific), according to the manufacturer's protocol. RNA samples were treated with Turbo DNase (Thermo Fisher Scientific) for $30 \mathrm{~min}$ at $37^{\circ} \mathrm{C}$. The RNA was reverse transcribed using the Superscript IV first-strand synthesis system (Thermo Fisher Scientific), according to the manufacturer's protocol. The resulting cDNAs were subjected to PCR amplification using the following primers: hBmal1b-F (5'-GCTCCACTGACTACCAAGA AAG-3'), hBmal1a-F (5'-GCTCCACTGACTACC ATTCACA-3'), and hBmal1-R (5'-GCTGAACAGCCA TCCTTAGC-3').

For qPCR, $1 \mu \mathrm{g}$ of DNase-treated RNA was reverse transcribed using random hexamers and Superscript IV reverse transcriptase. The level of each mRNA was normalized to Gapdh as an internal control, and the result was plotted as a normalized fold-change versus the control. Primers used for qPCR were: Gapdh-F (5'-TGCAC CACCAACTGCTTAGC-3'), Gapdh-R (5'-GCAGT GATGGCATGGACTGT-3'), hBmal1a-F (5'-CAAAGAT GACCCTCATGGAAG-3'), hBmalla-R (5'-CTTCCAT GAGGGTCATCTTTG-3'), hBmal1b-F (5'-TCCACTG ACTACCAAGAAAGCA-3'), hBmal1b-R (5'-ACTGTG AGCTTCCCTTGCAT-3'), hPer2-F (5'-CCAGTAAC CCCACCAAGGAG-3'), and hPer2-R (5'-ACTGCTC ATGTCCACATCTTCC-3').

\section{Nucleocytoplasmic fractionation}

HeLa, WI-38, or Myc-hBmal1-expressing HeLa cells were harvested in buffer A (50 mM HEPES, pH 7.4, 1.5 $\mathrm{mM} \mathrm{MgCl}$, and $10 \mathrm{mM} \mathrm{KCl}$ ) and incubated on ice for 15 min. After centrifugation at $3,000 \mathrm{rpm}$ for $10 \mathrm{~min}$ at $4{ }^{\circ} \mathrm{C}$, the supernatant was transferred to a new tube. For cytoplasmic fraction, the supernatant was centrifuged at $12,000 \mathrm{rpm}$ for $10 \mathrm{~min}$ at $4{ }^{\circ} \mathrm{C}$ and added to $4 \times$ sample buffer. For nuclear fraction, the pellet was resuspended in buffer C (50 mM HEPES, pH 7.4, $1.5 \mathrm{mM} \mathrm{MgCl}_{2}, 10 \mathrm{mM}$ $\mathrm{KCl}$, and $450 \mathrm{mM} \mathrm{NaCl}$ ) and incubated on ice for $30 \mathrm{~min}$. Samples were centrifuged at $12,000 \mathrm{rpm}$ for $10 \mathrm{~min}$ at $4{ }^{\circ} \mathrm{C}$ and the supernatants were collected. Fractionation was confirmed by examining the expression of Lamin B and $\beta$ Tubulin in the nuclear and the cytoplasmic fractions, respectively.

\section{Immunoprecipitation and western blotting}

HeLa cells were transfected with Flag-tagged hBmal1b and an increasing amount of Myc-tagged hBmalla. After an incubation for $48 \mathrm{~h}$, cells were lysed with $500 \mu \mathrm{l}$ of IP buffer [50 mM HEPES, pH 7.4, $150 \mathrm{mM} \mathrm{NaCl}, 1 \% \mathrm{NP}-40$,
$1 \mathrm{mM}$ EDTA, $1 \mathrm{mM}$ EGTA, $1 \mathrm{mM}$ PMSF, $0.5 \% \mathrm{Na}-$ deoxycholate and a protease inhibitor cocktail (SigmaAldrich, St. Louis, MO, USA)] and centrifuged at 12,000 rpm for $20 \mathrm{~min}$ at $4{ }^{\circ} \mathrm{C}$. After quantification by the Bradford assay, lysates were incubated with $2 \mu \mathrm{g}$ of anti-Flag antibody for $2 \mathrm{~h}$ at $4{ }^{\circ} \mathrm{C}$ and then incubated with a protein G-sepharose bead slurry for $1 \mathrm{~h}$ at $4{ }^{\circ} \mathrm{C}$. The final immune complexes were eluted with $2 \times$ sodium dodecyl sulfate (SDS) sample buffer and analyzed by western blotting.

For western blotting, protein samples were separated on 6\% SDS-polyacrylamide gels and transferred to PVDF membranes. Western blotting was performed with antiMyc (9E10; Santa Cruz Biotechnology, Dallas, TX, USA), anti-Flag (M2; Sigma-Aldrich), anti-CLOCK (S19; Santa Cruz Biotechnology), anti-BMAL1 (Novus Biologicals, Littleton, CO, USA), or anti-BMAL1 ${ }^{22}$ antibodies and HRP-conjugated secondary antibodies for enhanced chemiluminescence detection.

\section{Bimolecular fluorescence complementation assay and immunofluorescence}

The bimolecular fluorescence complementation (BiFC) assay was performed as described elsewhere ${ }^{23}$. Briefly, the $\mathrm{N}$-terminal 173 amino acid residues of the YFP (YN) and the C-terminal amino acid residues 173-238 of YFP (YC) were used to prepare fusion gene constructs for use in the BiFC assay. For the dual-color BiFC assay ${ }^{24}$, the partial sequences of CFP encoding amino acid residues 1-173 (CN) or amino acid residues 173-238 (CC) were used to construct the fusion genes. Fusion gene plasmids were transfected into HeLa cells. Twenty-four hours after transfection, cells were fixed with $4 \%$ paraformaldehyde in phosphate-buffered saline (PBS). After $1 \mathrm{~h}$ of blocking with $5 \%$ donkey serum and $0.3 \%$ Triton X-100 in PBS at room temperature, fixed cells were incubated with the indicated primary antibodies overnight at $4{ }^{\circ} \mathrm{C}$. Cells were then washed with PBS and incubated with fluorescent dye-conjugated secondary antibodies for $1 \mathrm{~h}$ at room temperature. Samples were analyzed by confocal microscopy (Nikon, Tokyo, Japan).

\section{Chromatin immunoprecipitation assay}

Myc-tagged hBmallb was transfected with an increasing amount of Flag-hBmalla into HeLa cells. Forty-eight hours after transfection, cells were fixed with $1 \%$ paraformaldehyde for $10 \mathrm{~min}$ at $37^{\circ} \mathrm{C}$. Cells were incubated with Lysis buffer (50 mM Tris- $\mathrm{HCl}, \mathrm{pH} 8.0,150 \mathrm{mM}$ $\mathrm{NaCl}, 2 \%$ Triton X-100, $10 \mathrm{mM}$ EDTA, and 1\% SDS) for $10 \mathrm{~min}$ at $4{ }^{\circ} \mathrm{C}$ and then sonicated to shear the chromatin. After centrifugation at $12,000 \mathrm{rpm}$ for $10 \mathrm{~min}$ at $4{ }^{\circ} \mathrm{C}$, the supernatant was transferred into new tubes and was diluted 10-fold with Dilution buffer $(50 \mathrm{mM}$ Tris- $\mathrm{HCl}, \mathrm{pH}$ 8.0, $150 \mathrm{mM} \mathrm{NaCl}, 0.1 \%$ Triton X-100, $10 \mathrm{mM}$ EDTA, and $1 \mathrm{mM}$ PMSF). Each cross-linked sample was 
immunoprecipitated with $2 \mu \mathrm{g}$ of anti-CLOCK (S19; Santa Cruz Biotechnology) or anti-Myc (9E10; Santa Cruz Biotechnology) antibodies at $4{ }^{\circ} \mathrm{C}$ overnight. Then, protein Gsepharose beads were added to each tube and incubated for $2 \mathrm{~h}$ at $4{ }^{\circ} \mathrm{C}$. The pellet was washed with low salt buffer $(20 \mathrm{mM}$ Tris- $\mathrm{HCl}, \mathrm{pH} 8.0,150 \mathrm{mM} \mathrm{NaCl}, 1 \%$ Triton X100, $2 \mathrm{mM}$ EDTA, and 0.1\% SDS), high salt buffer (20 mM Tris- $\mathrm{HCl}, \mathrm{pH}$ 8.0, $500 \mathrm{mM} \mathrm{NaCl}, 1 \%$ Triton X-100, $2 \mathrm{mM}$ EDTA, and $0.1 \%$ SDS), $\mathrm{LiCl}$ buffer (10 mM Tris-HCl, pH 8.0, $250 \mathrm{mM} \mathrm{LiCl}, 1 \% \mathrm{NP}-40,1 \mathrm{mM}$ EDTA, and $1 \% \mathrm{Na}-$ deoxycholate), and TE buffer ( $10 \mathrm{mM}$ Tris- $\mathrm{HCl}, \mathrm{pH} 8.0$, and $1 \mathrm{mM}$ EDTA). Samples were eluted with Elution buffer (1\% SDS and $0.1 \mathrm{M} \mathrm{NaHCO}_{3}$ ) and incubated with $0.2 \mathrm{M} \mathrm{NaCl}$ for $6 \mathrm{~h}$ at $65^{\circ} \mathrm{C}$ to reverse the cross-linking. Then, DNA was purified by phenol-chloroform extraction and ethanol precipitation. For PCR amplification of the proximal hPer1 E-box, the following primers were used: F (5'-GGAGGAAAGTACTAGACACCACGTA-3') and R (5'-CTCCCAACCTTCGTTCTACATAAT-3').

\section{Real-time monitoring of clock gene promoter expression}

For the real-time monitoring of circadian rhythmicity, NIH-3T3 cells were transfected with the hPer2-promoter conjugated to destabilized firefly luciferase together with $\mathrm{hBmal1 \textrm {a }}$ and/or hBmal1b, as indicated. When transfected cells reached confluence, $1 \mu \mathrm{M}$ dexamethasone (Dex; Sigma-Aldrich) was applied for $2 \mathrm{~h}$ to induce and synchronize the circadian rhythms. Real-time luciferase activity was measured using Kronos-Dio (Atto, Tokyo, Japan) for $72 \mathrm{~h}$.

\section{Generation of CRISPR/Cas9-mediated hBmal1a knockout cells}

Genome editing for hBmal1a knockout was performed as previously described ${ }^{25}$. The sgRNA constructs designed to skip exon 6 were inserted into the pSpCas9(BB)-2APuro (PX459) plasmid. The guide sequences were: sgRNA-i5 (5'-ATTTGGGTAAGATTCCACGC-3') and sgRNA-i6 (5'-TTAGCGCTTGCTCAAGACA A-3').

After transfection of sgRNA constructs, HeLa cells were incubated for $72 \mathrm{~h}$ and genomic DNA was extracted using the QuickExtract DNA extraction solution. The genomic region containing exon 6 of hBmall gene was amplified using the following primers: $F$ (5'-TGGAAGGAAT GAGTGGAGGT- $\left.3^{\prime}\right)$ and $\mathrm{R}$ (5'-GGCCAGGTGA TAATCTCAGG-3')

Heteroduplex DNA was obtained by the denaturation and renaturation of genomic PCR samples and then quantified using the T7 Endonucelase1 (T7E1) assay (New England Biolabs, Ipswich, MA, USA).

HeLa cells stably expressing sgRNA-i 5 and/or sgRNA-i6 were selected with puromycin. Genomic PCR and DNA sequencing were performed to detect the specific cells in which exon 6 of Bmall was deleted. RNA was isolated from HeLa cells or sgRNA-expressing cells and reverse transcribed using the Superscript IV first-strand synthesis system (Thermo Fisher Scientific) to confirm the expression of hBmalla mRNA. RT-PCR was performed using the following primer sequences: hBmal1-exon4-F (5'-TGAAAATCGCTTTGAGGTGA-3'), hBmalla-exon 6-F (5'-GGTCAGATGCCCACTAGGAG-3'), hBmal1bexon5\&7-F (5'-GCTCCACTGACTACCAAGAAAG-3'), and hBmal1-exon9-R (5'- GCTGAACAGCCATCCTT AGC-3').

\section{Statistical analysis}

The data are presented as the means \pm SE. The statistical significance of differences between groups was determined using one-way ANOVA. $P<0.01$ was regarded as a significant difference.

\section{Results}

\section{Expression of $h B m a l 1 a$ in human cell lines}

First, the expression of hBmal1a and hBmallb mRNAs was analyzed with quantitative PCR (qPCR) using the designated primers (Fig. 1a). As shown in Fig. 1b, a significant amount of hBmalla mRNA was detected in different human cell lines. However, the expression of a Bmal1 isoform corresponding to hBmalla was not detected in the mouse cell line NIH-3T3 and different mouse tissues (Supplementary Fig. S1b). The expression of a substantial amount of hBmal1a mRNA was also validated by RT-PCR (Supplementary Fig. S1c and d). Next, we examined the circadian expression profiles of $h B m a l 1 a, h B m a l 1 b$, and $h P e r 2$, a negative regulator of the molecular clock in cultured WI-38 human fibroblasts. Upon application of $50 \%$ horse serum for $2 \mathrm{~h}, \mathrm{hPer} 2$ and hBmallb mRNAs exhibited an anti-phasic circadian expression pattern for up to $48 \mathrm{~h}$, consistent with previous reports $^{26-28}$ (Fig. 1c). As shown in Fig. 1c, the expression profile of hBmalla mRNA was similar to hBmal1b.

Total lysates obtained from the human cell lines WI-38, HeLa, and HEK293T were subjected to western blotting using an anti-BMAL1 antibody to analyze the expression of endogenous hBMAL1a and hBMAL1 $b^{22}$. As shown in Fig. 1d, two distinct BMAL1 bands were detected in all three cell lines. Considering the difference in the sizes of hBMAL1b $(68.8 \mathrm{kDa})$ and hBMAL1a $(64.2 \mathrm{kDa})$, the upper (arrow) and lower (arrowhead) bands were predicted to represent hBMAL1b and hBMAL1a, respectively (Fig. 1d). Moreover, the lower band was not detected in the lysates of NIH-3T3 cells (Fig. 1d). In an effort to validate whether the lower band indeed corresponded to hBMAL1a, plasmids expressing hBmal1a or hBmal1b were transfected into Bmal1 knockout mouse embryonic fibroblasts $\left(\mathrm{MEF}^{\text {Bmal1-/-}}\right.$ ) in which no BMAL1 band was detected in western blotting (Fig. 1d, lanes 1-3). The difference in the sizes of exogenously expressed 

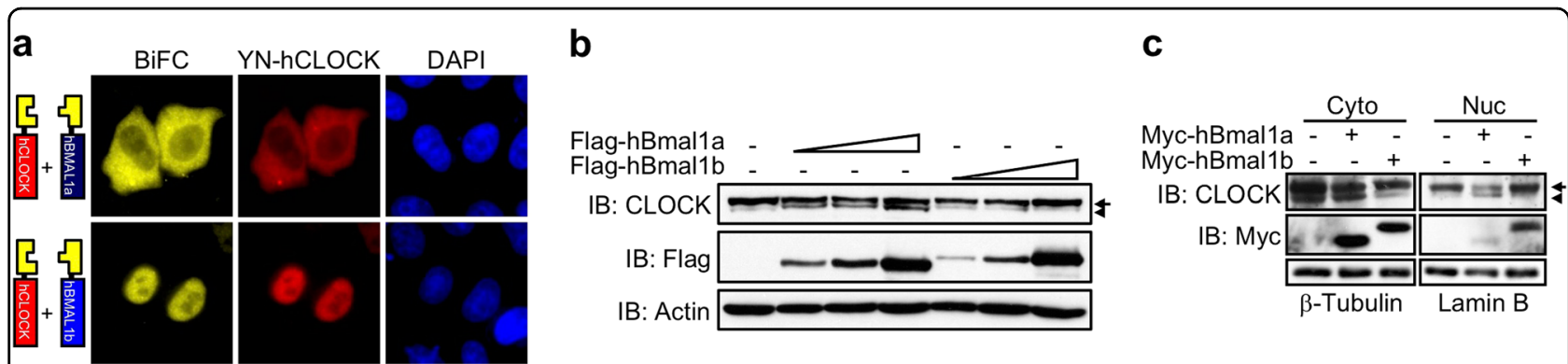

Fig. 2 Cytoplasmic retention of hCLOCK by hBMAL1a. a Direct interactions between hBMAL1 isoforms and hCLOCK were visualized by the BiFC assay. YN-hClock was co-transfected with YC-hBmal1a or YC-hBmal1b into HeLa cells. The subcellular localization of hCLOCK was detected by immunofluorescence staining using an anti-CLOCK antibody. $\mathbf{b}$ The expression of the endogenous hCLOCK was determined by western blotting in the presence of different levels of either Flag-hBMAL1a or hBMAL1b. c HeLa cells transfected with either Myc-hBmal1a or Myc-hBmal1b were subjected to nucleocytoplasmic fractionation. The level of hCLOCK in each fraction was analyzed by western blotting using an anti-CLOCK antibody

hBMAL1a and hBMAL1b was similar to the difference in the size of the two endogenous protein bands detected using the BMAL1 antibody (Fig. 1d). A proteasome inhibitor, MG132, had no effect on the intensity of the two BMAL1 bands, suggesting that the lower band is not a degraded form of hBMAL1b (Fig. 1d). BMAL1 was detected as hypo- or hyper-phosphorylated form on western blotting in the previous study ${ }^{26}$. To test the possibility that the lower BMAL1 band is the hypo- or unphosphorylated form of the hBMAL1b, a pull-down assay was performed following the application of phosphatases. As shown in Supplementary Fig. S2a-c, phosphatases had no effect on the mobility of both endogenous and exogenously expressed BMAL1 protein bands.

Due to the absence of the NLS, Myc-tagged hBMAL1a predominantly localized in the cytoplasm, while Myctagged hBMAL1b entered nuclei (Fig. 1e). In the western blotting, exogenously expressed Flag-tagged hBMAL1a was detected in both cytoplasmic and nuclear fractions, while Myc-tagged hBMAL1b was predominantly detected in the nuclear lysates (Supplementary Fig. S2d). When different human cell lysates were subjected to western blotting with an anti-BMAL1 antibody after nucleocytoplasmic fractionation, the lower BMAL1a band was predominantly detected in the cytoplasmic fraction, while the upper band was predominantly detected in the nuclear fraction (Fig. 1f).

\section{hBMAL1a-mediated cytoplasmic retention of hCLOCK}

Although the NLS is missing, hBMAL1a still possesses intact basic helix-loop-helix (bHLH) and PER-ARNTSIM (PAS) domains that are required for the interaction with its binding partner hCLOCK ${ }^{22}$. Additionally, nuclear entry of the CLOCK:BMAL1 complex is dependent on the NLS of BMAL1 ${ }^{22}$. Thus, hBMAL1a is presumed to form a complex with hCLOCK in the cytoplasm and thereby prevent hCLOCK from entering the nucleus. To visualize this possibility, the direct interaction between the two different hBMAL1 isoforms and hCLOCK was analyzed by BiFC assay ${ }^{9,23}$. In consistent with the previous report ${ }^{22}$ and as expected, the YFP signal representing the direct interaction between YFP $\mathrm{N}$-terminus (YN)-tagged hCLOCK (YN-hCLOCK) and YFP C-terminus (YC)-tagged hBMAL1b (YC-hBMAL1b) was detected in the nuclei (Fig. 2a, bottom panels), while the YFP signal of YNhCLOCK and YC-hBMAL1a complex was predominant in the cytoplasm (Fig. 2a, top panels).

When analyzed by western blotting, two differently sized hCLOCK proteins were detected (Fig. 2b, first lane). This finding is consistent with the previous report in which the electrophoretic mobility of CLOCK was altered by phosphorylation upon serum application to cultured cells ${ }^{29,30}$. Interestingly, the intensity of the fast-migrating lower band of hCLOCK was decreased by hBMAL1b in a dose-dependent manner (Fig. 2b, lanes 5-7), while the amount of the lower hCLOCK band was increased in the presence of hBMAL1a (Fig. 2b, lanes 2-4). In the pull down assay, Myc-tagged hBMAL1a or hBMAL1b predominantly coprecipitated with the lower or upper bands of hCLOCK, respectively (Supplementary Fig. S3). To further analyze the effect of hBMAL1 isoforms on hCLOCK, nuclear or cytoplasmic fractions were subjected to western blotting in the presence of hBMAL1a or hBMAL1b. First, as shown in Fig. 2c, lanes 1 and 4, in samples without any exogenous hBMAL1 proteins, the upper band was the dominant form of hCLOCK in the nucleus, while both upper and lower bands were detected in the cytoplasm. In the presence of overexpressed hBMAL1a, the amount of upper band in the nucleus decreased, while no apparent change was observed in the cytoplasm (Fig. 2c, lanes 2 and 5). On the other hand, the amount of the lower hCLOCK band in the cytoplasm decreased, while the amount of the upper hCLOCK band in the nucleus increased upon overexpression of hBMAL1b (Fig. 2c, lanes 3 and 6). Based on these data, 
hBMAL1b promotes the nuclear entry of hCLOCK, while hBMAL1a prevents hCLOCK from entering the nucleus.

\section{hBMAL1a promotes the proteolysis of hBMAL1b}

Theoretically, hBMAL1a, which has no NLS, should be predominantly retained in the cytoplasm. However, as shown in Fig. 1e and Supplementary Fig. S2d, a significant amount of hBMAL1a was still detected in the nucleus. Thus, hBMAL1a might be transported into the nucleus by another binding partner. In the previous study, it was reported that the recombinant BMAL1 protein forms BMAL1:BMAL1 homodimer complex in vitro ${ }^{31}$. Therefore, we hypothesized that hBMAL1a might enter the nucleus in the form of homodimer with hBMAL1b. To test this hypothesis, YN-hBmalla and YC-hBmallb were co-expressed in cultured cell lines. As expected, YFP signal representing the hBMAL1a:hBMAL1b complex was mainly detected in the nuclei (Fig. 3a, left panel), while the immunofluorescence signal for YN-hBMAL1a was detected in both the nuclei and cytoplasm (Fig. 3a, middle panel).

In the course of studying the effects of hBMAL1a: hBMAL1b homodimer formation, hBMAL1a was observed to promote the proteolysis of hBMAL1b in a dose-dependent manner (Fig. 3b; Supplementary Fig. S4). However, hBMAL1b had no effect on the stability of hBMAL1a protein (Fig. 3c). Moreover, hBMAL1amediated degradation of hBMAL1b was reversed upon application of MG132, a potent inhibitor of the $26 \mathrm{~S}$ proteasome (Fig. 3d). These data are consistent with the previous report showing that the splice variants of human luteinizing hormone receptor control the stability of the other variants through a direct interaction ${ }^{32}$. Taken together, hBMAL1a directly binds hBMAL1b and thereby induces the proteolysis of hBMAL1b.

\section{hBMAL1a interferes with the formation of hCLOCK: hBMAL1b heterodimers}

Having observed that hBMAL1a formed complexes with either hBMAL1b or hCLOCK, we hypothesized that hBMAL1a might compete with hBMAL1b for heterodimer formation with hCLOCK. To test this hypothesis, a dual-color BiFC assay was performed using $\mathrm{N}$-terminal CFP (CN)-tagged hBmallb (CN-hBmal1b), C-terminal CFP (CC)-tagged hClock (CC-hClock), and YNhBmal1a ${ }^{9,23}$. In the absence of YN-hBMAL1a, CFP signals representing the hCLOCK:hBMAL1b complex were detected in the nuclei (Fig. 4a, top row). However, nuclear CFP signals were diminished while cytoplasmic YFP signals representing hCLOCK:hBMAL1a were increased following the co-expression of YN-hBMAL1a in a dosedependent manner (Fig. 4a, first and second columns). Simultaneously, cytoplasmic retention of hCLOCK was also dose-dependently increased by YN-hBMAL1a

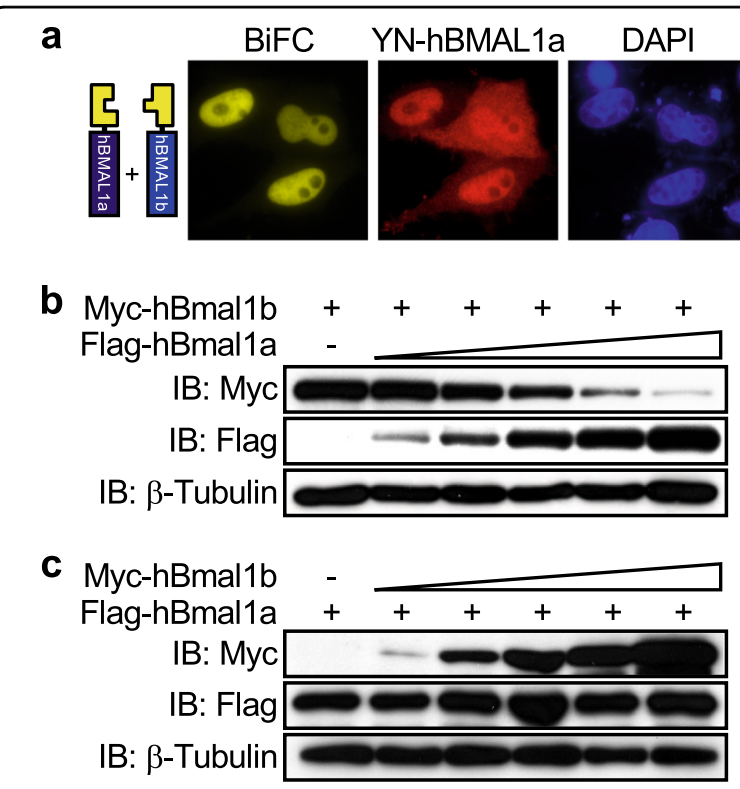

d

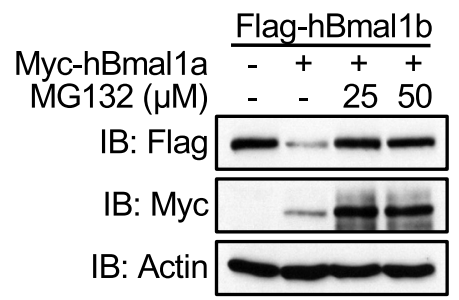

Fig. 3 hBMAL1a promotes the proteolysis of hBMAL1b. a The direct interaction between hBMAL1a and hBMAL1b was visualized by the BiFC assay using YN-Flag-fused hBmalla and YC-fused hBmal $1 \mathrm{~b}$. b, c Fixed amounts of Myc-hBmal1b or Flag-hBmal1a were cotransfected into HeLa cells with increasing amounts of Flag-hBmal1a or Myc-hBmal1b, respectively. Levels of Myc-hBMAL1a or FlaghBMAL1b were detected by western blotting. d Flag-hBmal1b was transfected alone or together with Myc-hBmal1a into HeLa cells. After an overnight incubation, a potent protease inhibitor, MG132, was applied to the transfected cells as indicated. After $5 \mathrm{~h}$ incubation, levels of hBMAL1 isoforms were analyzed by western blotting

(Fig. 4a, third column). Consistent with these findings, pull down of endogenous hCLOCK by Myc-tagged hBMAL1b was dose-dependently decreased by the coexpression of Flag-tagged hBMAL1a (Fig. 4b).

For the activation of downstream core clock genes, heterodimers of CLOCK:BMAL1 must be recruited to the E-box cis-elements in the promoter region of downstream genes $^{30}$. A chromatin immunoprecipitation assay was performed to determine whether hBMAL1a inhibits the recruitment of either hCLOCK or hBMAL1b to the E-box element. In the absence of hBMAL1a, both hCLOCK and exogenously expressed Myc-hBMAL1b were effectively recruited to the proximal E-box of the Period1 promoter (Fig. 4c, first lane). However, the E-box recruitment of hCLOCK or Myc-hBMAL1b was decreased by FlaghBMAL1a in a dose-dependent manner (Fig. 4c, second 


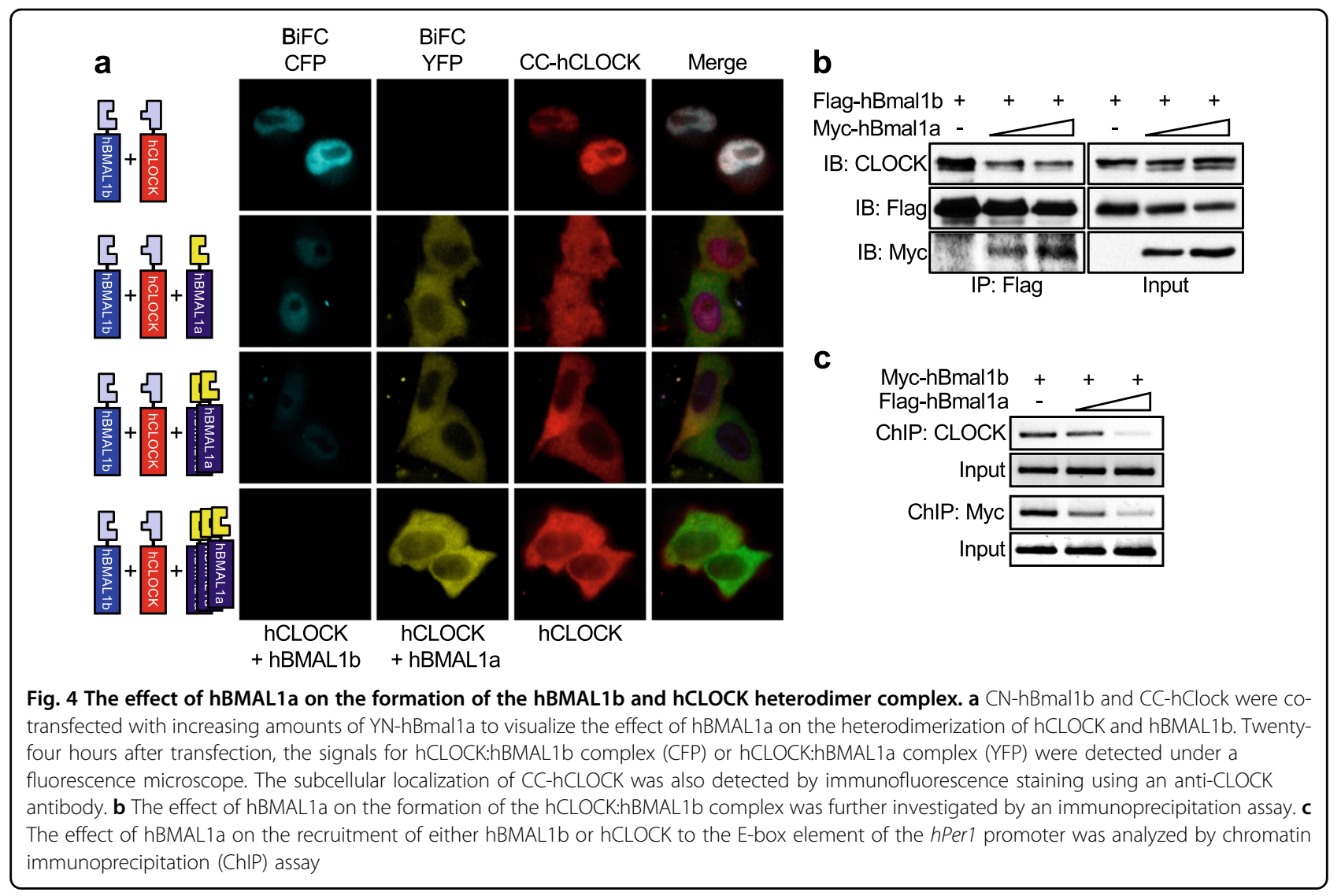

and third lanes). Taken together, these data suggest the evidence that hBMAL1a interferes with the formation and subsequent E-box recruitment of hCLOCK:hBMAL1b heterodimers.

\section{hBMAL1a functions as a negative regulator of the molecular clock}

Knowing that hBMAL1a interferes with the formation of the functional transcriptional activator complex between hCLOCK and hBMAL1b, we evaluated the effect of hBMAL1a on the transcriptional activation mediated by hCLOCK:hBMAL1b heterodimers. In a reporter assay using human Period1 (hPer1) promoter conjugated to the firefly Luciferase (hPer1-Luc), hBMAL1b and hCLOCKinduced transcriptional activation was attenuated by coexpression of hBMAL1a in a dose-dependent manner (Fig. 5a; Supplementary Fig. S5). To examine the effect of hBMAL1a on circadian rhythmic expression of clock gene, transcriptional activation of hPer2-dsLuc was monitored in NIH-3T3 cell line in real time for $72 \mathrm{~h}$ in the absence or presence of hBMAL1 isoforms. As shown in Fig. 5b, both the amplitude and period of the oscillatory expression of the $h P e r 2$ promoter was drastically reduced by hBMAL1a (red line), but not by hBMAL1b (blue line). Interestingly, co-expression of hBMAL1a and hBMAL1b exerted a greater inhibitory effect on the amplitude of hPer2 expression (Fig. 5b, green line).

To further evaluate the effect of hBMAL1a as a negative regulator of the molecular circadian clock, we generated a HeLa cell line in which expression of hBmalla was specifically abolished by CRISPR/Cas9-mediated knockout while hBmal1b expression was intact (Supplementary Fig. S6a-e). Upon application of $50 \%$ horse serum for $2 \mathrm{~h}$, the expression profiles of $h P e r 2, h B m a l 1 b$, and $h B m a l 1 a$ mRNAs were assessed by qPCR for $48 \mathrm{~h}$ at $4 \mathrm{~h}$ intervals in normal or hBmal1a knockout $\left(\mathrm{HeLa}^{\text {Bmalla-/-}}{ }^{-}\right.$) HeLa cells. As shown in Fig. $5 c$, d, the amplitude of $h B m a l 1 b$ and $h$ Per 2 expression was increased in $\mathrm{HeLa}^{\text {Bmalla-/- }}$ cells compared to normal HeLa cells. All together, these data offer the possibility that hBMAL1a might function as a negative regulator in the control of the human molecular clock.

\section{Discussion}

Here, we report evidence that the human-specific isoform of BMAL1 may function as a negative regulator of the molecular circadian clock. BMAL1, along with its binding partner CLOCK, is an indispensable core clock component that is required for the transcriptional activation of downstream clock genes. Moreover, as 

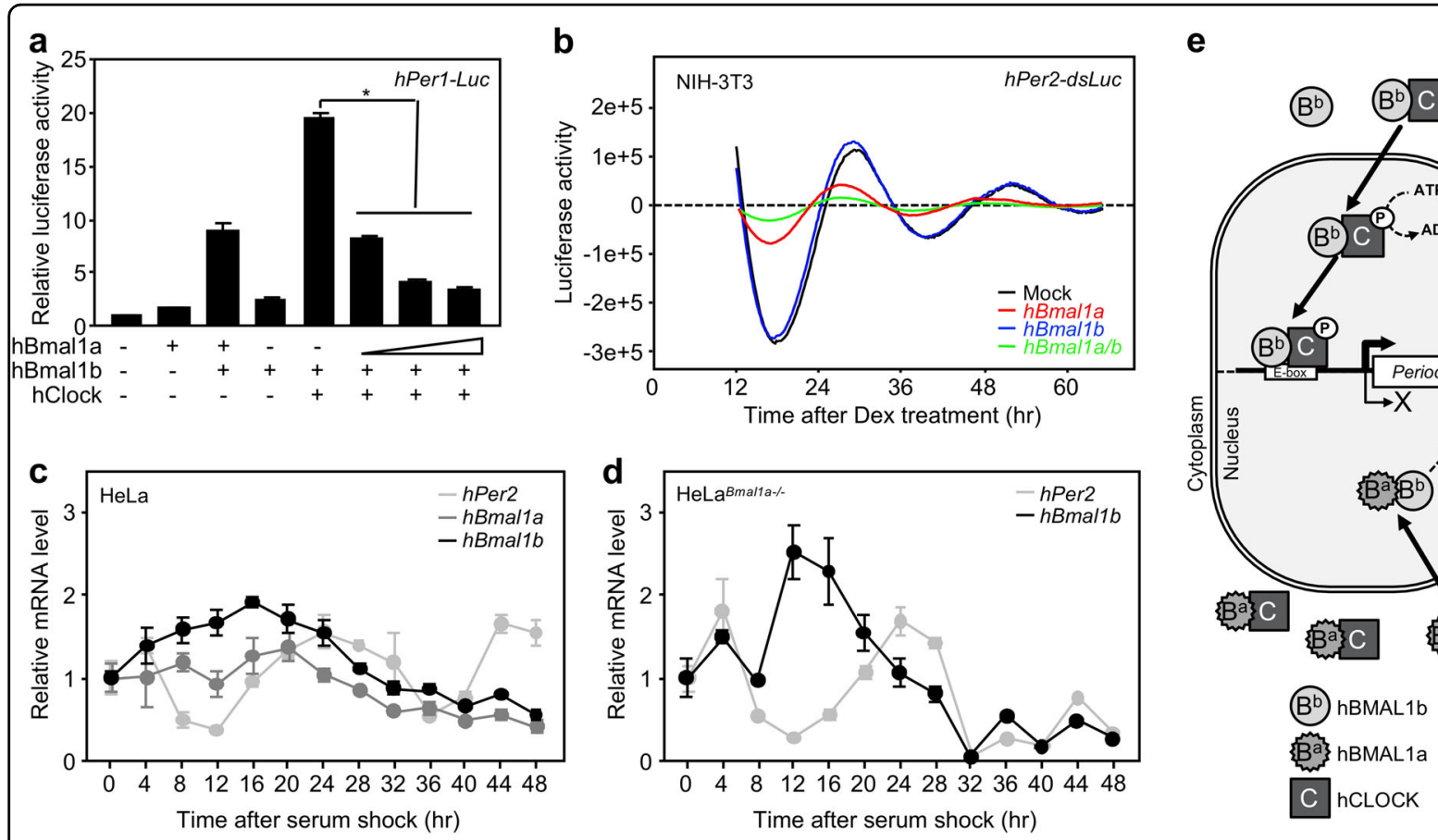

Fig. 5 Dominant-negative effect of hBMAL1a on the regulation of the molecular circadian clock. a The activation of hPer 1 promoter linked to firefly luciferase was assayed in HEK293T cells transfected with the indicated plasmid DNAs. The relative luciferase activities are shown as the means \pm standard errors $($ S.E. $)\left({ }^{*} P<0.01\right)$. b The effect of hBMAL1a and/or hBMAL $1 \mathrm{~b}$ on the rhythmic expression profile of the $h$ Per2 promoter linked to destabilized firefly luciferase (dsLuc) was measured in NIH-3T3 cells for $72 \mathrm{~h}$ using Kronos-Dio (Atto). c, d Circadian expression levels of $h B m a l 1 a$, hBmal $1 b$, and $h$ Per2 mRNAs in HeLa or HeLa ${ }^{B m a l l a-/-}$ cells were analyzed. Following the application of $50 \%$ horse serum for $2 \mathrm{~h}$, relative mRNA levels were determined by qPCR for $48 \mathrm{~h}$. e A model for the dominant-negative function of hBMAL1a. In the cytoplasm, hBMAL1a interferes with the formation of hCLOCK:hBMAL1b heterodimer complex by binding hCLOCK or hBMAL1b proteins. Through this direct interaction, hBMAL1a prevents hCLOCK phosphorylation and nuclear translocation, which are closely related to the transcriptional activity of hCLOCK. The hBMAL1a protein also promotes the proteolysis of hBMAL1b through a direct interaction. Consequently, hBMAL1a functions as a dominant-negative regulator in the control of the molecular circadian clock

shown in our previous studies, we have reported that the nuclear entry of the CLOCK:BMAL1 heterodimer is solely dependent on the NLS of BMAL1 ${ }^{21,22}$. Thus, we reasonably presumed that the NLS-deficient hBMAL1a might exert a negative effect on the transcriptional activation mediated by canonical hBMAL1b and hCLOCK complex. Indeed, hBMAL1a effectively inhibited the transcriptional activation of hPer1 promoter in a luciferase reporter assay (Fig. 5a; Supplementary Fig. S5). Furthermore, the amplitude of the oscillatory expression of hPer2 was suppressed in the presence of hBMAL1a (Fig. 5b).

Based on our results, hBMAL1a may directly interact with hCLOCK or hBMAL1b in the course of inhibiting the transcriptional activation of downstream clock genes. The electrophoretic mobility of CLOCK protein differs depending on its phosphorylation status ${ }^{29,30}$. In mice, CLOCK mainly exists as a hypo-phosphorylated form throughout the day, while hyper-phosphorylated CLOCK is detected only at the phase of repression ${ }^{29}$. The level of the unphosphorylated CLOCK protein begins to decrease at the time of the transcriptional activation of the downstream clock gene $D b p^{29}$. Based on these previous observations, it is presumed that the upper and lower bands of human CLOCK in the present study represented the hypo- and unphosphorylated forms, respectively (Fig. 2b). First of all, hypophosphorylated hCLOCK is predominantly located in the nucleus, while both un- and hypo-phosphorylated forms exist in the cytoplasm (Fig. 2c, first and fourth lanes). As shown in Fig. 2b, hBMAL1b promotes an upward shift of the unphosphorylated form of hCLOCK (Fig. 2b, lanes 5-7). Simultaneously, nuclear entry of the hypo-phosphorylated form of hCLOCK was increased in the presence of exogenously expressed hBMAL1b (Fig. 2c, sixth lane). On the other hand, overexpression of hBMAL1a resulted in both the cytoplasmic retention of hCLOCK and an increase in the level of the unphosphorylated form of hCLOCK (Fig. 2a, b, lanes 5-7). These data suggest that hBMAL1b promotes both the phosphorylation and nuclear entry of hCLOCK (Fig. 5e, top half), while hBMAL1a prevents hCLOCK 
phosphorylation and nuclear translocation (Fig. 5e, bottom half).

Unexpectedly, we detected the unphosphorylated form of hCLOCK in the nuclear fraction in the presence of overexpressed hBMAL1a (Fig. 2c, fifth lane). As revealed by the BiFC assay, hBMAL1a was delivered into the nucleus by hBMAL1b (Fig. 3a). Moreover, hBMAL1a promoted the degradation of hBMAL1b in both the nucleus and cytoplasm (Fig. 3b; Supplementary Fig. S4a). Once delivered into the nucleus by hBMAL1b, hBMAL1a may dissociate from the complex as soon as it promotes the degradation of bound hBMAL1b. The free nuclear hBMAL1a then competes with its canonical isoform, hBMAL1b, for binding to hCLOCK in the nucleus. Actually, a portion of hCLOCK:hBMAL1a dimers was observed in the nuclei in the BiFC assay (Fig. 2a, top panels). Therefore, hBMAL1a might be able to promote the dephosphorylation of hCLOCK through a direct interaction in the nucleus.

A hBmal1a-specific knockout HeLa cell line was established to evaluate the physiological role of hBMAL1a. In the present study, rhythmic expression profiles of hPer2, hBmal1b, and hBmal1a mRNAs in HeLa cells were not as robust as in the normal human fibroblasts (Figs. 5c and 1c). However, the rhythmicity of $h$ Per2 and hBmallb mRNA expression was somewhat restored in the hBmalla knockout HeLa cells (Fig. 5d). Together, the data presented in this study indicate that hBMAL1a functions as a dominant-negative regulator of the molecular circadian clock. Studies of the dominantnegative effects of the splice variants of the transcription factors have been reported previously. For example, one of the isoforms of AFX (Foxo4), a member of the forkhead transcription factor family, is known to suppress the transcriptional activation of downstream genes by its canonical isoform in a dose-dependent manner ${ }^{33}$. The same pattern of dominant-negative regulation of transcription was reported for the transcription factors STAT3 or SND1 $1^{34,35}$.

In conclusion, we are the first to show that a splice variant of human BMAL1 functions as a dominantnegative regulator of transcription. Interestingly, despite the similarity in the genomic sequence around exon 6 of the Bmal1 gene in humans (exon 5-intron-exon 6-intron-exon 7) and mice (exon 5-intron-exon 6), the alternative splicing of exon 6 is observed only in humans (Fig. 1a). According to recent genomics studies, alternative RNA splicing patterns are not generally conserved in the human and mouse genomes, while most of the exons are strongly conserved in both genomes ${ }^{36}$. Taken together, we propose that the human-specific isoform of BMAL1 might be responsible for another level of finetuning in the control of human molecular circadian clock.

\section{Acknowledgements}

This work was supported by the National Research Foundation of Korea (NRF) grant (NRF-2017R1D1A1B03032080) awarded to J.L., the NRF grant (NRF2016R1C1B2008776) awarded to I.K., and the NRF grant (NRF-

2017R1A2A1A05001351) and the DGIST MIREBraiN Programs (2017010095 and 2017040002) awarded to K.K. by the Korean government.

\section{Author details}

${ }^{1}$ Department of Anatomy and Cell Biology, Sungkyunkwan University School of Medicine, Suwon 16419, Korea. ${ }^{2}$ GenWay Biotech, Inc., 6777 Nancy Ridge Dr, San Diego, CA 92121, USA. ${ }^{3}$ Department of Brain and Cognitive Sciences, Daegu Gyeongbuk Institute of Science and Technology (DGIST), Daegu 42988, Korea

\section{Conflict of interest}

The authors declare that they have no conflict of interest.

\section{Publisher's note}

Springer Nature remains neutral with regard to jurisdictional claims in published maps and institutional affiliations.

Supplementary information accompanies this paper at https://doi.org/ 10.1038/s12276-018-0187-X.

Received: 2 May 2018 Revised: 10 September 2018 Accepted: 12 September 2018.

Published online: 6 December 2018

\section{References}

1. Harbison, C. T. et al. Transcriptional regulatory code of a eukaryotic genome. Nature 431, 99-104 (2004).

2. Schibler, U. Circadian time keeping: the daily ups and downs of genes, cells, and organisms. Prog. Brain Res. 153, 271-282 (2006).

3. Gallego, M. \& Virshup, D. M. Post-translational modifications regulate the ticking of the circadian clock. Nat. Rev. Mol. Cell Biol. 8, 139-148 (2007).

4. Gekakis, N. et al. Role of the CLOCK protein in the mammalian circadian mechanism. Science 280, 1564-1569 (1998).

5. Hogenesch, J. B., Gu, Y. Z., Jain, S. \& Bradfield, C. A. The basic-helix-loop-helixPAS orphan MOP3 forms transcriptionally active complexes with circadian and hypoxia factors. Proc. Natl. Acad. Sci. USA 95, 5474-5479 (1998).

6. Jin, X. et al. A molecular mechanism regulating rhythmic output from the suprachiasmatic circadian clock. Cell 96, 57-68 (1999).

7. Reppert, S. M. \& Weaver, D. R. Molecular analysis of mammalian circadian rhythms. Annu. Rev. Physiol. 63, 647-676 (2001).

8. Hirayama, J. et al. CLOCK-mediated acetylation of BMAL1 controls circadian function. Nature 450, 1086-1090 (2007).

9. Lee, J. et al. Dual modification of BMAL1 by SUMO2/3 and ubiquitin promotes circadian activation of the CLOCK/BMAL1 complex. Mol. Cell Biol. 28, 6056-6065 (2008)

10. Lowrey, P. L. \& Takahashi, J. S. Genetics of the mammalian circadian system: photic entrainment, circadian pacemaker mechanisms, and posttranslational regulation. Annu. Rev. Genet. 34, 533-562 (2000).

11. Gossan, N. C. et al. The E3 ubiquitin ligase UBE3A is an integral component of the molecular circadian clock through regulating the BMAL1 transcription factor. Nucleic Acids Res. 42, 5765-5775 (2014).

12. Chen, S. et al. Ubiquitin-conjugating enzyme UBE2O regulates cellular clock function by promoting the degradation of the transcription factor BMAL1. J. Biol. Chem. 293, 11296-11309 (2018).

13. Collett, M. A., Dunlap, J. C. \& Loros, J. J. Circadian clock-specific roles for the light response protein WHITE COLLAR-2. Mol. Cell Biol. 21, 2619-2628 (2001).

14. Diernfellner, A. et al. Long and short isoforms of Neurospora clock protein FRQ support temperature-compensated circadian rhythms. FEBS Lett. 581, 5759-5764 (2007)

15. Diernfellner, A. C., Schafmeier, T., Merrow, M. W. \& Brunner, M. Molecular mechanism of temperature sensing by the circadian clock of Neurospora crassa. Genes Dev. 19, 1968-1973 (2005). 
16. Allada, R., Kadener, S., Nandakumar, N. \& Rosbash, M. A recessive mutant of Drosophila Clock reveals a role in circadian rhythm amplitude. EMBO J. 22 3367-3375 (2003)

17. Majercak, J., Sidote, D., Hardin, P. E. \& Edery, I. How a circadian clock adapts to seasonal decreases in temperature and day length. Neuron 24, 219-230 (1999).

18. Ikeda, M. \& Nomura, M. cDNA cloning and tissue-specific expression of a novel basic helix-loop-helix/PAS protein (BMAL1) and identification of alternatively spliced variants with alternative translation initiation site usage. Biochem. Biophys. Res. Commun. 233, 258-264 (1997).

19. $\mathrm{Yu}, \mathrm{W}$. et al. Characterization of three splice variants and genomic organization of the mouse BMAL1 gene. Biochem. Biophys. Res. Commun. 260, 760-767 (1999).

20. Wolting, C. D. \& McGlade, C. J. Cloning and chromosomal localization of a new member of the bHLH/PAS transcription factor family. Mamm. Genome $\mathbf{9}$ 463-468 (1998).

21. Kondratov, R. V. et al. BMAL1-dependent circadian oscillation of nuclear CLOCK: posttranslational events induced by dimerization of transcriptional activators of the mammalian clock system. Genes Dev. 17, 1921-1932 (2003).

22. Kwon, I. et al. BMAL1 shuttling controls transactivation and degradation of the CLOCK/BMAL1 heterodimer. Mol. Cell Biol. 26, 7318-7330 (2006).

23. Hu, C. D., Chinenov, Y. \& Kerppola, T. K. Visualization of interactions among bZIP and Rel family proteins in living cells using bimolecular fluorescence complementation. Mol. Cell 9, 789-798 (2002).

24. Hu, C. D. \& Kerppola, T. K. Simultaneous visualization of multiple protein interactions in living cells using multicolor fluorescence complementation analysis. Nat. Biotechnol. 21, 539-545 (2003).

25. Ran, F. A. et al. Genome engineering using the CRISPR-Cas9 system. Nat. Protoc. 8, 2281-2308 (2013).
26. Lee, C., Etchegaray, J. P., Cagampang, F. R., Loudon, A. S. \& Reppert, S. M Posttranslational mechanisms regulate the mammalian circadian clock. Cell 107, 855-867 (2001).

27. Miyazaki, K. et al. Phosphorylation of clock protein PER1 regulates its circadian degradation in normal human fibroblasts. Biochem. J. 380, 95-103 (2004).

28. Balsalobre, A., Damiola, F. \& Schibler, U. A serum shock induces circadian gene expression in mammalian tissue culture cells. Cell 93, 929-937 (1998).

29. Yoshitane, $\mathrm{H}$. et al. Roles of CLOCK phosphorylation in suppression of E-boxdependent transcription. Mol. Cell Biol. 29, 3675-3686 (2009).

30. Shim, H. S. et al. Rapid activation of CLOCK by Ca2+-dependent protein kinase $\mathrm{C}$ mediates resetting of the mammalian circadian clock. EMBO Rep. $\mathbf{8}$, 366-371 (2007).

31. Rutter, J., Reick, M., Wu, L. C. \& McKnight, S. L. Regulation of clock and NPAS2 DNA binding by the redox state of NAD cofactors. Science 293, 510-514 (2001).

32. Nakamura, K., Yamashita, S., Omori, Y. \& Minegishi, T. A splice variant of the human luteinizing hormone $(\mathrm{LH})$ receptor modulates the expression of wildtype human LH receptor. Mol. Endocrinol. 18, 1461-1470 (2004).

33. Lee, E. J., Kim, J. M., Lee, M. K. \& Jameson, J. L. Splice variants of the forkhead box protein AFX exhibit dominant negative activity and inhibit AFXalphamediated tumor cell apoptosis. PLOS ONE 3, e2743 (2008).

34. Caldenhoven, E. et al. STAT3beta, a splice variant of transcription factor STAT3, is a dominant negative regulator of transcription. J. Biol. Chem. 271, 13221-13227 (1996).

35. Li, Q. et al. Splice variant of the SND1 transcription factor is a dominant negative of SND1 members and their regulation in Populus trichocarpa. Proc Natl. Acad. Sci. USA 109, 14699-14704 (2012).

36. Modrek, B. \& Lee, C. J. Alternative splicing in the human, mouse and rat genomes is associated with an increased frequency of exon creation and/or loss. Nat. Genet. 34, 177-180 (2003). 